\title{
Interdependent Values without Single-Crossing [Abstract]
}

\author{
ALON EDEN, Tel-Aviv University \\ MICHAL FELDMAN, Tel-Aviv University and Microsoft Research \\ AMOS FIAT, Tel-Aviv University \\ KIRA GOLDNER, University of Washington
}

\begin{abstract}
We consider a setting where an auctioneer sells a single item to $n$ potential agents with interdependent values. That is, each agent has her own private signal, and the valuation of each agent is a known function of all $n$ private signals. This captures settings such as valuations for oil drilling rights, broadcast rights, pieces of art, and many more.

In the interdependent value setting, all previous work has assumed a so-called single-crossing condition. Single-crossing means that the impact of a private signal, $s_{i}$, on the valuation of agent $i$, is greater than the impact of $s_{i}$ on the valuation of any other agent. It is known that without the single-crossing condition, an efficient outcome cannot be obtained. We ask what approximation to the optimal social welfare can be obtained when valuations do not exhibit single-crossing.

We show that, in general, without the single-crossing condition, one cannot hope to approximate the optimal social welfare any better than assigning the item to a random bidder. Consequently, we consider a relaxed version of single-crossing, $c$-single-crossing, with some parameter $c \geq 1$, which means that the impact of $s_{i}$ on the valuation of agent $i$ is at least $1 / c$ times the impact of $s_{i}$ on the valuation of any other agent $(c=1$ is single-crossing). Using this relaxed notion, we obtain a host of positive results. These include a prior-free universally truthful $2 \sqrt{n} c^{3 / 2}$-approximation to welfare, and a prior-free deterministic $(n-1) c$-approximation to welfare. Under appropriate concavity conditions, we improve this to a prior-free universally truthful $2 c$ approximation to welfare as well as a universally truthful $O\left(c^{2}\right)$-approximation to the optimal revenue.
\end{abstract}

\section{CCS Concepts: $\bullet$ Theory of computation $\rightarrow$ Algorithmic game theory and mechanism design;}

Additional Key Words and Phrases: Interdependent values; single-crossing; welfare maximization; prior-free

\footnotetext{
A full version of this paper is available at https://www.cs.tau.ac.il/ alonarde/interdependent.pdf.

Author's email addresses: alonarden@gmail.com; michal.feldman@cs.tau.ac.il; fiat@tau.ac.il; kgoldner@cs.washington.edu.

The work of A. Eden, M. Feldman, and K. Goldner was partially supported by the European Research Council under the European Union's Seventh Framework Programme (FP7/2007-2013) / ERC grant agreement number 337122, and by the Israel Science Foundation (grant number 317/17). The work of A. Eden and A. Fiat was partially supported by ISF 1841/14. The work of K. Goldner was supported in part by NSF award CCF-1420381 and by a Microsoft Research PhD Fellowship.

Permission to make digital or hard copies of part or all of this work for personal or classroom use is granted without fee provided that copies are not made or distributed for profit or commercial advantage and that copies bear this notice and the full citation on the first page. Copyrights for third-party components of this work must be honored. For all other uses, contact the owner/author(s).
}

ACM EC'18, fune 18-22, 2018, Ithaca, NY, USA.

(C) 2018 Copyright held by the owner/author(s)

ACM ISBN 978-1-4503-4529-3/18/06.

https://doi.org/10.1145/3219166.3219173 